\title{
A case report of allergic bronchopulmonary mycosis caused by Alternaria alternata in colonization of Aspergillus
}

\author{
Min Ju Jo, ${ }^{1 *}$ Yoomi Yeo, ${ }^{1 *}$ Kyueng-Whan Min, ${ }^{2}$ Sung Jun Chung, ${ }^{1}$ Tai Sun Park, ${ }^{1}$ Hyun Lee, ${ }^{1}$ Dong Won Park, ${ }^{1}$ \\ Ji-Yong Moon, ${ }^{1}$ Jang Won Sohn, ${ }^{1}$ Sang-Heon Kim, ${ }^{1}$ Ho Joo Yoon, ${ }^{1}$ Tae-Hyung Kim ${ }^{1}$
}

\begin{abstract}
Background: Allergic bronchopulmonary aspergillosis (ABPA) is a pulmonary disease caused by a complex hypersensitivity reaction to colonization of the airways with various fungi. ABPA caused by Alternaria alternata, other than Aspergillus spp., is named Allergic bronchopulmonary mycosis (ABPM).
\end{abstract}

Objective: To describe the first case of ABPM caused by Alternaria alternata in East Asia.

Methods: Case report

Results: A 58-year-old female visited our hospital due to an abnormal chest x-ray, following chest computed tomography (CT) revealed consolidation in the left lower lobe. On laboratory finding, eosinophil count and total IgE level were high. The skin prick test and specific IgE for Alternaria alternata were positive. After diagnosis of ABPM, the patient was treated with prednisolone without antifungal agents, and her chest image was much improved.

Conclusion: Aspergillus is most common etiology of allergic pulmonary disease, however, Alternaria should be considered even though positive culture of Aspergillus spp.

Key words: ABPM, Allergic bronchopulmonary, Alternaria alternate, Asthma, Specific IgE

\footnotetext{
From:

${ }^{1}$ Division of Pulmonology, Department of Internal Medicine, Hanyang University College of Medicine, Seoul, Republic of Korea

2 Department of Pathology, Hanyang University Guri Hospital, Hanyang University College of Medicine, Seoul, Republic of Korea

* These authors contributed equally to this work.
}

\section{Introduction}

Allergic bronchopulmonary aspergillosis (ABPA) is a pulmonary disease caused by a complex hypersensitivity reaction to colonization of the airways with various fungi, such as $A s-$ pergillus spp. ${ }^{1}$ It predominantly occurs in patients with asthma or cystic fibrosis. ${ }^{2}$ The disease is clinically characterized by recurrent infiltrations on chest radiograph, peripheral blood eosinophilia, elevated Immunoglobulin E (IgE) level in the serum, positive fungus-specific IgE and/or Immunoglobulin G (IgG) antibodies, and central bronchiectasis.,

Other than Aspergillus spp., other fungi reported to cause ABPA include Candida albicans, Alternaria, Bipolaris, Penicillium, and Trichosporon. Disease caused by these fungi is called Allergic bronchopulmonary mycosis (ABPM). ${ }^{5}$ Alternaria alternata, a ubiquitous saprophytic fungus found mostly in soil

\section{Corresponding author:}

Tae-Hyung Kim

Division of Pulmonology, Department of Internal Medicine,

Hanyang University College of Medicine,

222 Wangsimni-ro, Seongdong-gu, Seoul 04763, Korea

E-mail: drterry@hanyang.ac.kr

and plants, has been reported as the etiology of allergic rhinosinusitis, bronchial asthma, hypersensitivity pneumonitis, occulomycosis, onychomycosis, and skin infection. However, it typically does not cause ABPM, with only one case reported by Chowdhary et al. in $2012 .{ }^{5}$

There are no universally established diagnostic criteria for ABPM, but the most commonly used criteria are essentially the same as those used for ABPA. The diagnostic criteria proposed by Rosenberg et al. ${ }^{6}$ in 1977 were commonly used until the ABPA criteria were re-established in 2012. ${ }^{7}$

Here, we report a rare case of ABPM caused by Alternaria alternata in an immunocompetent patient as the first case in East Asia. 


\section{Case report}

A 58-year-old female visited our outpatient clinic of pulmonology division at Hanyang University Guri Hospital in South Korea due to an abnormal chest $\mathrm{x}$-ray obtained on a routine exam. She had been admitted for post-influenza pneumonia 3 years prior and suffered from chronic cough and intermittent bloody sputum but had never visited other clinics for further evaluation. She did not have fever, chest pain, and weight loss. She was a never smoker, and had no previous history of allergic disease, rhinitis, asthma, bronchiectasis, and tuberculosis. She was diagnosed as hypertension and diabetes 15 years ago, which were well-controlled with medications.

Laboratory investigations of white blood cell count, platelet count, random blood sugar, and renal and liver function tests were within the normal ranges as defined for her age. Abnormal findings were as follows: low hemoglobin, $9.8 \mathrm{~g} /$ dl; differential count, $10.2 \%$ eosinophils (absolute eosinophil count $[\mathrm{AEC}], 7,650 \mathrm{cells} / \mu \mathrm{l})$; and high erythrocyte sedimentation rate (ESR), $108 \mathrm{~mm} / \mathrm{hr}$ rather than normal range of C-reactive protein (CRP). A stool test for parasites (including ova) and blood cultures were negative, as were fluorescent antinuclear antibody (FANA), c-anti-neutrophil cytoplasmic antibody (ANCA) and p-ANCA performed to investigate vasculitis.

On chest x-ray, pulmonary infiltration was observed in the left lower lung field. Chest computed tomography (CT) revealed mass-like lesions with low attenuation in the left lower lung (LLL). Despite treatment with the moxifloxacin $400 \mathrm{mg} /$ day for seven days for community-acquired pneumonia, serial chest $\mathrm{x}$-rays demonstrated poor improvement (Figure 1A and 1B).

After check the poor improvement of CXR on day 4 of hospitalization, flexible bronchoscopy with bronchoalveolar lavage (BAL) under moderate sedation was planned for a microbiologic study. In the anterior segment of the LLL, 3 aliquots of $100 \mathrm{~mL}$ normal saline were administered using a gentle suction of $60 \mathrm{~mm} \mathrm{Hg}$, which led to a $45 \%$ return. Smears revealed inflammatory cells with marked macrophages along with a few scattered eosinophils (7\%) and lymphocytes (5\%). However, Aspergillus spp. was positive in fungal culture a few weeks after bronchoscopy. Pulmonary function test showed a normal flow pattern with a negative bronchodilator test and a negative methacholine provocation test (forced expiratory volume in 1 second $\left(\mathrm{FEV}_{1}\right)$ /forced vital capacity (FVC) $83 \%$, $\mathrm{FEV}_{1}$ was 2.78 liter, 104\% predicted and FVC was 3.35 liter, $101 \%$ predicted).

Two months later, the chest CT and serologic laboratory results were re-evaluated. The chest CT continued to show consolidation in the LLL (Figure 1C). The eosinophil level was lower compared to baseline but still high at 1,200/ $\mathrm{mm}^{3}$, and total IgE level via chemiluminescent immunoassay (CLIA) also was high at $1,280 \mathrm{IU} / \mathrm{mL}$. Therefore, allergic pulmonary disease was suspected.

The skin prick test and specific IgE for Alternaria alternata $(28.1 \mathrm{IU} / \mathrm{mL}$; normal range, $<0.2 \mathrm{IU} / \mathrm{mL})$ via fluoroenzyme immunoassay (FEIA) were positive. On the other hand, these two tests for aspergillus were negative. The patient fit all the diagnostic criteria for ABPM, such as immediate skin reactivity, high total serum IgE (over $416 \mathrm{IU} / \mathrm{mL}$ ), increased specific IgE antibodies, and subacute clinical deterioration, although she was not asthmatic.

Additionally, the patient underwent percutaneous lung biopsy that showed layers of mucin and inflammatory cells at low power. High power showed the characteristic "allergic" mucin, which was pale blue and intermixed with numerous eosinophils. Also, Charcot-Leyden crystals were seen in the allergic mucin under high power (Figure 2).

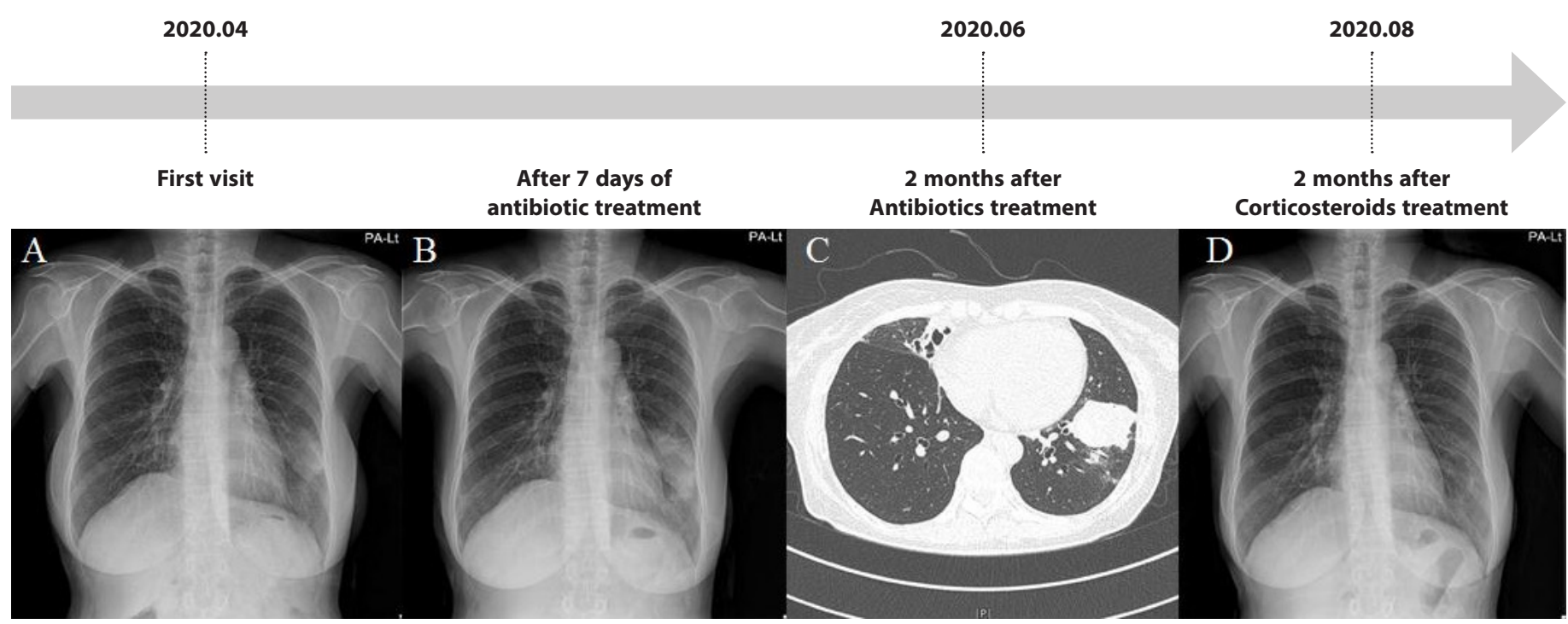

Figure 1. (A) Posteroanterior chest radiographs of the case patient at first visit. (B) Chest radiograph shows aggravated pulmonary infiltrates. (After treatment of antibiotics, day 7) (C) Chest computed tomography scan at 2 months after medical treatment. It shows a sustained consolidation in the left lower lobe. (D) Chest radiograph at the 2 months after medical treatment using corticosteroids shows much improvement of patch opacity of the lesion. 


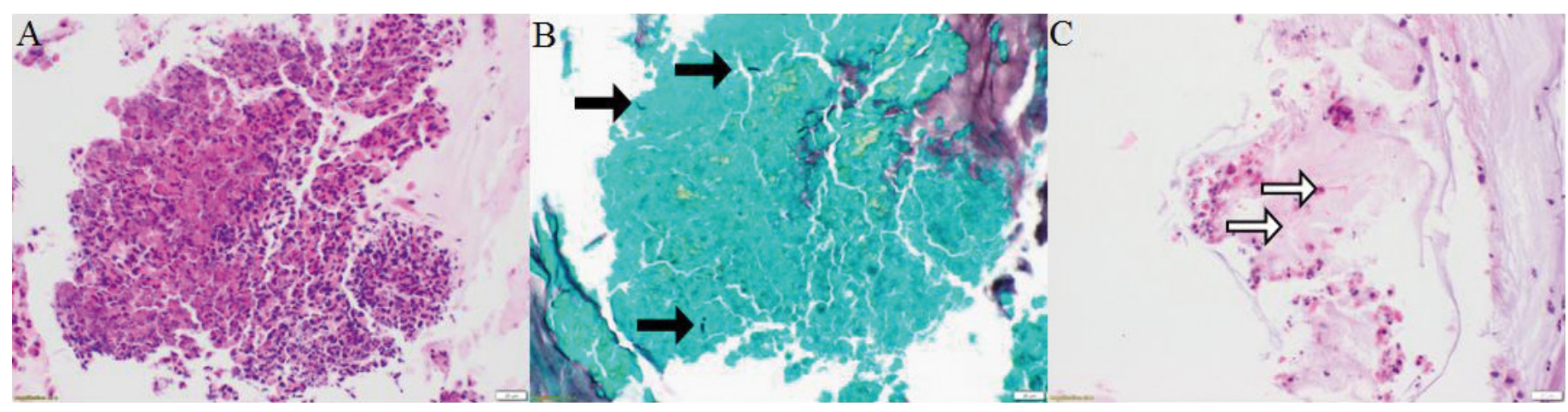

Figure 2. Histopathologic findings in percutaneous lung biopsy of the lesion. (A) Layers of mucin and inflammatory cells are shown in the low-power field in the plug removed. (B) The high-power filed shows the characteristic "allergic" mucin (black arrows), which is pale blue and intermixed with numerous eosinophils. (C) Charcot-Leyden crystals (white arrows) are shown in the high-power field of allergic mucin.

After diagnosis of ABPM, the patient received prednisolone $30 \mathrm{mg} /$ day without antifungal agents. Two weeks later, the chest $\mathrm{x}$-ray improved, the serum eosinophil count decreased (Figure 1D), and her symptoms resolved. After systemic corticosteroid treatment, the eosinophil level was 174/ $\mathrm{mm}^{3}$ and the total serum IgE level was $531 \mathrm{IU} / \mathrm{mL}$.

\section{Discussion}

Since its first description by Hinson et al. in 1952, ABPM due to fungi or yeast has been described in case reports. ${ }^{8}$ However, only one case of ABPM by Alternaria alternata was reported, by Chowdhary et al. ${ }^{5}$ in 2012, and there have been no reports in East Asia. The case patient showed similar clinical features to ABPA and cultures of BAL fluid positive for Aspergillus spp. However, allergic tests, including skin tests or serology tests and allergen-specific IgE via the FEIA method, were all negative to Aspergillus fumigatus.

Although there is no globally recognized set of diagnostic criteria for ABPM, the diagnostic criteria for ABPA are used by replacing Aspergillus fumigatus with other various fungi for diagnosis of ABPM.

In 1977, Rosenberg and Patterson proposed the diagnostic criteria for ABPA, which include 7 categories. However, the criteria for ABPA were re-established in a review in 2012, ${ }^{9}$ as follows: 1) asthma or cystic fibrosis with deterioration of lung function, 2) immediate Aspergillus species skin test reactivity, 3) total serum IgE level over $416 \mathrm{IU} / \mathrm{mL}$, 4) increased Aspergillus species-specific IgE and IgG antibodies, and 5) chest radiograph infiltrates. Additional criteria include peripheral blood eosinophilia, Aspergillus species serum precipitating antibodies, central bronchiectasis, and Aspergillus species containing mucus plugs.

There are several methods to measure total serum IgE and serum allergen-specific IgE they include the radioimmunoassay (RIA) method using isotopes and enzyme immynoassay (EIA) method using an enzyme. Enzyme-linked immunoassay (ELISA) is the common detection method of EIA. FEIA, CLIA and electrochemiluminescent (ECLIA) methods are types of modified ELISA method. ${ }^{10}$ They have high sensitivity and specificity compared to the skin prick test. ${ }^{11}$ The results of allergen-specific IgE tests in serum are consistent with the results of a skin prick test. As the results of the two tests provide different information, it is recommended to perform both if ABPM is suspected. ${ }^{11}$

In our case, total serum IgE level was measured by both the ECLIA method and the CLIA method, and the results were positive in both methods: $1,280 \mathrm{IU} / \mathrm{mL}$ via ECLIA and $1,716 \mathrm{IU} / \mathrm{mL}$ via CLIA. Both of these values are higher than the cut off value of the diagnostic criteria. In addition, serum-specific IgE level, measured by the FEIA method, of $A l$ ternaria alternata was $28.1 \mathrm{IU} / \mathrm{mL}$, corresponding to Phadia IgE class IV. In the skin prick test for Alternaria, a positive finding was confirmed as $3+$. On the contrary, the skin prick test for Aspergillus spp. was negative, and the allergen-specific IgE level was low at $2.09 \mathrm{IU} / \mathrm{mL}$. Combining these results, the patient was diagnosed with ABPM due to Alternaria alternate, and the Aspergillus in culture was suspected to have come from colonization in the bronchus.

The only diagnostic criterion that does not fit in this patient's case is the presence of asthma or cystic fibrosis, which is essential for diagnosis in ABPA. However, this is the case of ABPM and the diagnostic criteria have not been clearly outlined so far, because of few reported case.

To our knowledge, this is the first case of ABPM caused by Alternaria alternata in East Asia, and the second case worldwide. This is significant because ABPM occurred in a patient without asthma. Patients with ABPM usually have asthma, and it would be easy to overlook ABPM in patients without asthma. Therefore, this case suggests that ABPM should be considered in patients with symptoms suspicious for allergic respiratory disease incompatible with asthma.

In conclusion, we described a rare case of ABPM in a patient without asthma. When evaluating patients with symptoms of allergic respiratory disease, physicians should consider ABPM. In addition, total IgE and serum allergen-specific IgE should be measured and the skin prick test should be performed in patients whose symptoms and evaluations suggest ABPM

\section{Ethics statement}

Patient consent was obtained for both clinical data and images. 


\section{Conflict of interest}

On behalf of all authors, the corresponding author states that there is no conflict of interest.

\section{Source of funding}

None. No funding to declare.

\section{Author Contributions}

- Diagnosis: Yeo Y.

- Writing - original draft preparation: Jo MJ,

- Histological review: Min KH,

- Review and editing: Kim TH,

- Approval of final manuscript: all authors.

\section{References}

1. Al-Mobeireek AF, El-Rab M, Al-Hedaithy SS, Alasali K, Al-Majed S, Joharjy I. Allergic bronchopulmonary mycosis in patients with asthma: period prevalence at a university hospital in Saudi Arabia. Respir Med. 2001;95(5):341-7.

2. Asano K, Kamei K, Hebisawa A. Allergic bronchopulmonary mycosis - pathophysiology, histology, diagnosis, and treatment. Asia Pac Allergy. 2018;8(3):e24.
3. Chowdhary A, Agarwal K, Kathuria S, Gaur SN, Randhawa HS, Meis JF. Allergic bronchopulmonary mycosis due to fungi other than Aspergillus: a global overview. Crit Rev Microbiol. 2014;40(1):30-48.

4. Fukutomi Y, Tanimoto H, Yasueda H, Taniguchi M. Serological diagnosis of allergic bronchopulmonary mycosis: Progress and challenges. Allergol Int. 2016;65(1):30-6

5. Chowdhary A, Agarwal K, Randhawa HS, Kathuria S, Gaur SN, Najafzadeh $\mathrm{MJ}$, et al. A rare case of allergic bronchopulmonary mycosis caused by Alternaria alternata. Med Mycol. 2012;50(8):890-6.

6. Rosenberg M, Patterson R, Mintzer R, Cooper BJ, Roberts M, Harris $\mathrm{KE}$. Clinical and immunologic criteria for the diagnosis of allergic bronchopulmonary aspergillosis. Ann Intern Med. 1977;86(4):405-14.

7. Greenberger PA, Bush RK, Demain JG, Luong A, Slavin RG, Knutsen AP. Allergic bronchopulmonary aspergillosis. J Allergy Clin Immunol Pract. 2014;2(6):703-8.

8. Hinson KF, Moon AJ, Plummer NS. Broncho-pulmonary aspergillosis; a review and a report of eight new cases. Thorax. 1952;7(4):317-33.

9. Knutsen AP, Bush RK, Demain JG, Denning DW, Dixit A, Fairs A, et al. Fungi and allergic lower respiratory tract diseases. J Allergy Clin Immunol. 2012;129(2):280-91;quiz 92-3.

10. Zhang QY, Chen H, Lin Z, Lin JM. Comparison of chemiluminescence enzyme immunoassay based on magnetic microparticles with traditional colorimetric ELISA for the detection of serum alpha-fetoprotein. J Pharm Anal. 2012;2(2):130-5.

11. Chung HL. Clinical significance of serum IgE. Clin Exp Pediatr 2007;50(5):416-21. 